\title{
Evidence for both copy number and allelic (NA1/NA2) risk at the FCGR3B locus in systemic lupus erythematosus
}

\author{
David L Morris ${ }^{1}$, Amy L Roberts ${ }^{1}$, Abigail S Witherden ${ }^{1}$, Ruth Tarzi ${ }^{2}$, Paula Barros ${ }^{1}$, John C Whittaker ${ }^{3}$, \\ Terence H Cook ${ }^{4}$, Timothy J Aitman ${ }^{5}$ and Timothy J Vyse ${ }^{\star, 1}$
}

The Fc $\gamma$-receptor locus on chromosome 1q23 shows copy-number variation (CNV), and it has previously been shown that individuals with reduced numbers of copies of the Fc $\gamma$-receptor-IIIB gene (FCGR3B) have an increased risk of developing systemic lupus erythematosus (SLE). It is not understood whether the association arises from FCGR3B (CD16b) itself, is observed because of linkage disequilibrium with actual causal alleles and/or is an effect of CNV on flanking FCGR genes. Thus, we extended this previous work by genotyping the FCGR3B alleles NA1/NA2 and re-assaying CNV using a paralogue ratio test assay in a family study (365 families). We have developed a novel case/pseudo-control approach to analyse family data, as the phase of copy number (CN) is not known in parents and cannot always be inferred in offspring. The results, obtained by fitting logistic regression models, confirm the association of low $C N$ of $F C G R 3 B$ with SLE $(P=0.04)$. The risk conferred by low copies $(<2)$ was contingent on FCGR3B allotype, being greater for deletion of NA1 than the for lower-affinity NA2. The simpler model with just $C N$ was rejected in favour of the biallelic-CN model $(P=0.03)$. We observed a correlation $\left(R^{2}=0.75, P<0.0001\right)$ between FCGR3B CNV and neutrophil expression in both healthy controls and patients with SLE. Our results suggest that one mechanism by which CNV at this locus confers disease risk is directly as a result of reduced Fc $\gamma$ RIIIb function, either because of reduced expression (related to CNV) or because of reduced affinity for its ligand (NA1/NA2 allotype).

European Journal of Human Genetics (2010) 18, 1027-1031; doi:10.1038/ejhg.2010.56; published online 5 May 2010

Keywords: FCGR3B; NA1/NA2; genetics; systemic lupus erythematosus; CNV

\section{INTRODUCTION}

Systemic lupus erythematosus (SLE) is an autoimmune disease affecting multiple organ systems, which is characterized by the presence of autoreactive B cells and the formation of antibody-antigen immune complexes. The genetic contribution to SLE disease risk is strong; ${ }^{1-3}$ however, it is also a genetically complex disease. ${ }^{1-3}$ An increased understanding of the structure of the genome is leading to novel discoveries that will help unravel the mechanisms involved. ${ }^{4}$

Structural variation is recognized as a rich source of genetic heterogeneity in the human genome, and copy number variants (CNVs) account for a large part of this diversity. ${ }^{5-7}$ About half of the currently identified CNVs encompass genes, thus implicating $\mathrm{CNV}$ in disease pathogenesis. Thus far, variation in gene copy number $(\mathrm{CN})$ has been associated with a number of complex inflammatory and infectious disorders, ${ }^{8}$ including, although not without some controversy, ${ }^{9,10}$ HIV (CCL3L1), ${ }^{11}$ SLE (FCGR3B and complement C4) ${ }^{4,12,13}$ and Crohn's disease $\left(\right.$ IRGM) ${ }^{14}$

The FCGR region on chromosome 1q23.3 shows a complex pattern of $\mathrm{CNV}$ and sequence homology. From the five FCGR genes within the 172-kb region, three (FCGR3A, FCGR2C and FCGR3B) have been reported to show $\mathrm{CNV}^{15}$ although the frequency of $\mathrm{CNV}$ at FCGR3A is low.
FCGR genes encode functionally diverse Fc $\gamma$ - receptors, which recognise the Fc portion of immunoglobulin molecules. It is their specificity for different immunoglobulin isotopes and pattern of tissue expression that define the Fc $\gamma$ Rs. Fc $\gamma$ RIIIa, expressed on monocytes, macrophages and NK cells, and Fc $\gamma$ RIIIb expressed on neutrophils, are low-affinity activating receptors. This function is known to be altered in SLE patients, which makes the FCGR genes key candidates for disease susceptibility. Genetic associations have been reported between SLE and functional polymorphisms at FCGR2A, FCGR2B and FCGR3A. ${ }^{16-19}$ Also there have been studies reporting the association of two common allotypes of FCGR3B with SLE. ${ }^{20-22}$ However, the interpretation of these studies is bedevilled by the confounding effect of CNV of the FCGR3B gene.

An association between low FCGR3B CN and SLE in a UK case-control study was reported recently. ${ }^{4}$ However, there are issues regarding the reliability and accuracy of quantitative PCR (qPCR) to assay $\mathrm{CNV},{ }^{23}$ as was used in that study. In addition, it is not understood whether the association arises from FCGR3B itself, and/or is an effect of $\mathrm{CNV}$ on flanking FCGR genes. Thus, we extended this previous work by genotyping the FCGR3B alleles NA1/NA2 (HNA1a/HNA1b), and re-assaying $\mathrm{CNV}$, in a larger study using a family-based (365 families) approach, which should be robust to population stratification.

${ }^{1}$ Section of Rheumatology, Hammersmith Hospital, Imperial College London, London, UK; ${ }^{2}$ Department of Renal, Imperial College London, London, UK; ${ }^{3}$ Department of Epidemiology and Population Health, London School of Hygiene and Tropical Medicine, London, London, UK; ${ }^{4}$ Department of Histopathology, Imperial College London, London, UK; ${ }^{5} \mathrm{MRC}$ Clinical Sciences Centre, Hammersmith Hospital, Imperial College London, London, UK.

${ }^{\star}$ Correspondence: Professor TJ Vyse, Faculty of Medicine, Section of Molecular Genetics and Rheumatology, Imperial College London, Du Cane Road, London W12 ONN, UK. Tel: +44 208383 2339; Fax: +44 208383 2379; E-mail: t.vyse@imperial.ac.uk

Received 8 December 2009; revised 12 February 2010; accepted 17 February 2010; published online 5 May 2010 
CNV and relative CNs of the NA1 and NA2 alleles of FCGR3B were determined using a paralogue ratio test (PRT) assay. ${ }^{24,25}$ PRT is a PCR-based assay, ${ }^{25,26}$ which uses the high sequence identity of FCGR3A and FCGR3B to give accurate CN estimates for both. PRT returns $\mathrm{CN}$ measurements with more precision than qPCR because of simultaneous amplification of a target and a control. In addition, CN estimates from PRT are closer to integer values rather than continuous variables as in $\mathrm{qPCR}$, and PRT provides data on the NA1/NA2 allotype, which qPCR does not.

We applied the logistic regression case-control methodology to detect CNV and allotype effects, using an innovative case/pseudocontrol approach. In addition, we studied the relationship of both $\mathrm{CNV}$ at FCGR3B and the NA1/NA2 allotype with other genetic polymorphisms at the FCGR locus. Our results support an association between SLE and both CNV at FCRG3B and its allotype.

\section{MATERIALS AND METHODS}

\section{Study cohort}

The association of FCGR3B genotype with SLE was studied in 365 UK Caucasian SLE families ( 365 trios).

All families were recruited through UK rheumatology clinics or by direct patient contact following media publicity. Ethical approval was obtained through MREC98/2/06 and 06/MRE02/9, and all participants gave appropriate informed consent. Each proband met the ACR 1982 revised criteria for diagnosis. ${ }^{27}$ Clinical data and sera were collected at a single time point at study enrolment. Disease phenotype varied, but data collection from patients who were not infected (C-reactive protein $<15 \mathrm{mg} / \mathrm{l}$ ) or having a disease flare took place in an outpatient setting. The patients comprised 333 females and 32 males, all Caucasian. Mean age at diagnosis was 38.2 years ( $\mathrm{SD}=9.04$ years), with first and fourth quadrants of 32 and 44 years, respectively. Supplementary Table 1 contains more information on the sub-phenotypes.

\section{Paralogue ratio test}

$\mathrm{CN}$ values were obtained using a PCR-based PRT as described previously. ${ }^{24,25}$ The assay was run in duplicate on 96-well plates, each containing seven positive controls of varying CNs. Each experiment was calibrated using linear regression of the expected $\mathrm{CN}$ on the observed $\mathrm{CN}$ for the positive controls. Plates were repeated if the positive controls gave unexpected results.

\section{Restriction enzyme digest variant ratios}

A multiplex PCR was used to amplify two regions, which were then digested with Taq $\alpha 1$ (New England Biolabs, Ipswich, MA, USA). One restriction enzyme digest variant ratio was used to distinguish between FCGR3A and FCGR3B, and another to distinguish between NA1 and NA2 allotypes of FCGR3B as described previously. ${ }^{24}$ VIC (Applied Biosystems, Bedford, MA, USA) fluorescent label was used instead of HEX in the latter assay.

\section{$\mathrm{CN}$ estimates}

Fluorescent labelled (FAM and HEX) PCR products were pooled together and analysed by electrophoresis on an Applied Biosystems genetic analyser. Genemapper (Applied Biosystems) software was used to visually analyse the results. Products of 67 and $72 \mathrm{bp}$ were amplified from chromosome 1 and chromosome 18, respectively. Peak area ratios were used to estimate a total $\mathrm{CN}$ for FCGR3A and FCGR3B against the non-CNV region on chromosome 18. Mean values were taken from the duplicates and used in further analysis.

\section{Genotype estimates}

Fluorescent labelled (FAM and VIC; FAM and HEX) restriction enzyme digest variant ratio products were analysed by electrophoresis on an Applied Biosystems genetic analyser. Genemapper (Applied Biosystems) software was used to visually analyse the results. Peak areas at 134 (digested - FCGR3A) and $182 \mathrm{bp}$ (undigested - FCGR3B) were used to generate 3A:3B ratios. Peak areas at 174 (digested - NA1) and $209 \mathrm{bp}$ (undigested - NA2) were used to generate NA1:NA2 ratios.
Mean PRT values and both restriction enzyme digest variant ratios were used to estimate the $\mathrm{CN}$ values for FCGR3A and FCGR3B, and NA1/NA2 allotype at the FCGR3B locus, given that FCGR3A does not vary from the NA1 allotype. For example, a sample with $\mathrm{PRT}=4,3 \mathrm{~A}: 3 \mathrm{~B}=1$ and $\mathrm{NA} 1 \mathrm{NA} 2=1$ would be genotyped as $F C G R 3 A=2, F C G R 3 B=2, \mathrm{NA} 2 / \mathrm{NA} 2$.

There were no Mendelian errors observed for any of the 365 families with respect to $\mathrm{CN}$ or allotype at FCGR3B.

\section{Flow cytometry}

The antibodies used in this study were anti-Fc $\gamma$ RIII (clone 3G8; BD Biosciences, Oxford, UK), anti-Fc $\gamma$ RIII (clone LNK 16, Serotec, Kidlington, UK), antiFc $\gamma$ RIIIb (Clone 1D3 Serotec), anti-Fc $\gamma$ RII (clone FLI8.26; BD Biosciences) and anti-CD19 (BD Biosciences). Isotype controls were IgG2a (clone G155-178), $\operatorname{IgG} 1 \kappa$ (clone MOPC-21) and IgG2b $\kappa$ (clone MPC-11). Whole blood aliquots of volume $100 \mu \mathrm{l}$ were incubated with fluorescent labelled antibodies in the concentrations recommended by the manufacturer, for $15 \mathrm{~min}$ at room temperature. All patients gave informed consent for analysis of DNA and blood samples. Erythrocytes were lysed using BD FACS Lysing Solution, and surface expression assessed by flow cytometry. FITC and PE CaliBRITE beads were used to ensure that fluorescence settings on the FACS machine were stable. Granulocytes were selected on the basis of size and granularity. Data were analysed using FlowJo software (Tree Star, Inc., Ashland, OR, USA). Results were analysed using GraphPad Prism 3.0 (GraphPad Software, La Jolla, CA, USA).

\section{FCGR2B I232T genotyping (rs1050501)}

Amplification of $40 \mathrm{ng}$ of gDNA was carried out in a final volume of $20 \mu \mathrm{l}$, using 0.5U HotStar Taq Polymerase (Qiagen, Valencia, CA, USA), forward primer as described previously ${ }^{28}$ and reverse primer $5^{\prime}$-GCTTGGGTGGCC CCTGGTTCTCA- $3^{\prime}$. Owing to the high degree of homology between FCGR2B and FCGR2C, the reverse primer is located in intron 6 of FCGR2B, which is not present in FCGR2C. The conditions for amplification were an initial enzyme activation step of $95^{\circ} \mathrm{C}$ for $15 \mathrm{~min}$, followed by $94^{\circ} \mathrm{C}$ for $30 \mathrm{~s}, 60^{\circ} \mathrm{C}$ for $30 \mathrm{~s}$ and $72{ }^{\circ} \mathrm{C}$ for $2.5 \mathrm{~min}$ for $35 \mathrm{cycles}$, and a final $72{ }^{\circ} \mathrm{C}$ for $10 \mathrm{~min}$. PCR products were run on a $1.5 \%$ agarose gel. ExoSAP-IT ( $4 \mu \mathrm{l}$; GE Healthcare, Buckinghamshire, UK) was added to $10 \mu \mathrm{l}$ of the PCR product and incubated at $37^{\circ} \mathrm{C}$ for $15 \mathrm{~min}$ and at $80{ }^{\circ} \mathrm{C}$ for $15 \mathrm{~min}$. Products were sequenced on a 3730xl DNA Analyser using forward (5'-TGCCTGTCCTGATGTCTGTC- $\left.3^{\prime}\right)$ and reverse (5'-GGGCCAAGTGGAAACTGATA- $\left.3^{\prime}\right)$ primers, which are located closer to the I232T polymorphism in exon 5 . BioEdit was used to visually analyse the electropherograms.

\section{Statistical analysis}

Logistic regression analysis was used to model disease risk as a function of $\mathrm{CN}$ and allotype. With regard to $\mathrm{CN}$ this is simply an additive model, on the logistic scale, with $\mathrm{CN}(0-4$ in our data) as the explanatory variable.

For the allelic data we developed a novel approach to family analysis. The data for each subject consist of two variables: the quantity of observed NA1 and the quantity of observed NA2 (a three-copy genotype, NA1/NA1/NA2, would be [2 1]). We adopted an approach, that can be regarded as an extension of the transmission disequilibrium test ${ }^{29}$ approach. We matched each child with a 'pseudo-control' by deducting the observed quantities for the child from the total observed quantities in the parents. For example, if an affected child had genotype [NA1/NA1/NA2] with parents' genotypes being [NA1/NA2] and [NA1/NA2], then the pseudo-control would be a one-copy genotype NA2 with variables for our analysis of [0 1$]$. With the assumption of Mendelian inheritance, the matched genotypes [2 1$]$ and [ 01 1], given parents' genotypes as $\left[\begin{array}{ll}1 & 1\end{array}\right]$ and [ 111$]$, have equal probability of transmission under the null hypothesis that allotype does not affect the disease risk. This example assumes that one parent has a deletion on one chromosome and two copies on the other, with the two-copy haplotype transmitted (see Supplementary Figure 1). The other parent is assumed to have one copy on each chromosome. However, in many cases it is not possible to determine the phase. Nevertheless, a case and pseudo-control can always be made in the same way with equal transmission probabilities under the null. A lack of Mendelian inheritance would not lead to false-positive findings, as (under the null) there is no reason 
to believe that de novo events would favour increased/decreased NA1 or NA2 in offspring.

We adopted a 'bottom-up' approach to variable selection with regard to testing for $\mathrm{CN}$ effect and allelic effects. The $\mathrm{CN}$ model was tested first, and then the allelic model was tested against the $\mathrm{CN}$ model using a likelihood ratio test. ${ }^{30}$

To determine the correlation between the FCGR3B (NA1/NA2) locus and other SNPs, we calculated $R^{2}$ between SNP genotypes $(0,1,2)$ and the following variables:

a. For correlation with $\mathrm{CN}$; number of copies.

b. For correlation between alleles; two variables (NA1+NA2; multiple $R^{2}$ ).

We use method (b) to assess the correlation between alleles regardless of $\mathrm{CN}$ at FCGR3B (this incorporates cases with one copy). For both (a) and (b) we used independent data only (parents only). Significance for correlation between $F C G R 3 B$ and a SNP is assessed with reference to the $P$-value for the regression from where the $R^{2}$ arises.

We checked our results against the more standard estimates $\left(D^{\prime}\right.$ and $\left.R^{2}\right)$ of linkage disequilibrium using just the two-copy individuals, which included complete trios where possible.

To calculate the correlation between the 'null haplotype' (zero copy on a chromosome) for FCGR3B and SNPs, we took all individuals with either 0,1 or 2 copies and, taking the null haplotype as the 'minor allele', we coded the genotype as [ [1 1 $]$, [ [ 12 2], or [2 2 2], respectively. This assumes that all two-copy individuals have one copy on each chromosome. Standard measures of linkage disequilibrium such as $D^{\prime}$ and $R^{2}$ will capture any correlation.

The correlation analysis was performed using genotyping for FCGR2A and FCGR3A taken from an earlier study, ${ }^{31}$ and the genotyping for $F C G R 2 B$ as mentioned above.

The linkage disequilibrium analysis on two-copy individuals and the nullhaplotype analysis were done using Haploview. All other analyses were performed using $R$.

\section{RESULTS}

We found significant evidence for an association between $\mathrm{CN}$ and disease risk (Table $1 ; P=0.04$ ). The odds ratio of 0.71 implies a protective effect with increased copies (disease risk decreases by 0.71 for each additional copy). Furthermore, we found evidence of an allelic effect over and above the $\mathrm{CN}$ effect $(P=0.032)$. Looking at the odds ratios for NA1 and NA2 in Table 1, it is evident that NA1 has a stronger protective effect than NA2 (OR $=0.62$ and 0.78 for NA1 and NA2, respectively). Assessed on its own merits, without comparison with the $\mathrm{CN}$ model, this allelic model is significant ( $P=0.01$, null; genotype has no effect on disease risk). The fit of our allelic model to the data, along with the relative effects of NA1 and NA2, can be seen in Figure 1. The model cuts through the data very closely, and the gradient in the NA1 direction is much steeper than for NA2 (odds of the disease decreases by 0.62 for each copy of NA1

Table 1 Logistic regression results for copy number and allelic models

\begin{tabular}{lllcc}
\hline Model & Variable & P-value & OR & LRT P-value \\
\hline CN & No. of copies & 0.039 & $0.71(0.52,0.98)$ & \\
Allelic & NA1 & $0.011^{\mathrm{a}}$ & $0.62(0.44,0.87)$ & $0.032^{\mathrm{a}}$ \\
& NA2 & & $0.78(0.56,1.08)$ &
\end{tabular}

All models were subjected to logistic regression with case/control status as the outcome. The 'CN' model has the total number of copies as the explanatory variable. The 'allelic' model has two explanatory variables (number of copies of NA1 and number of copies of NA2) and therefore two parameter estimates and two odd ratios. The 'LRT $P$-value' column indicates the test of the allelic model against the CN model; small $P$-values imply that we reject the $\mathrm{CN}$ model in favour of the allelic model.

alndicates significance at the 0.05 level. compared with 0.78 for NA2). Frequencies for NA1 and CN can be seen in Table 2.

We found no evidence of an association between $\mathrm{CN}$ at FCGR3A and disease risk $(P=0.46)$, and no interaction between $\mathrm{CN}$ at $3 A$ and $3 B$ affecting the disease risk ( $P=0.89$ for interaction effect).
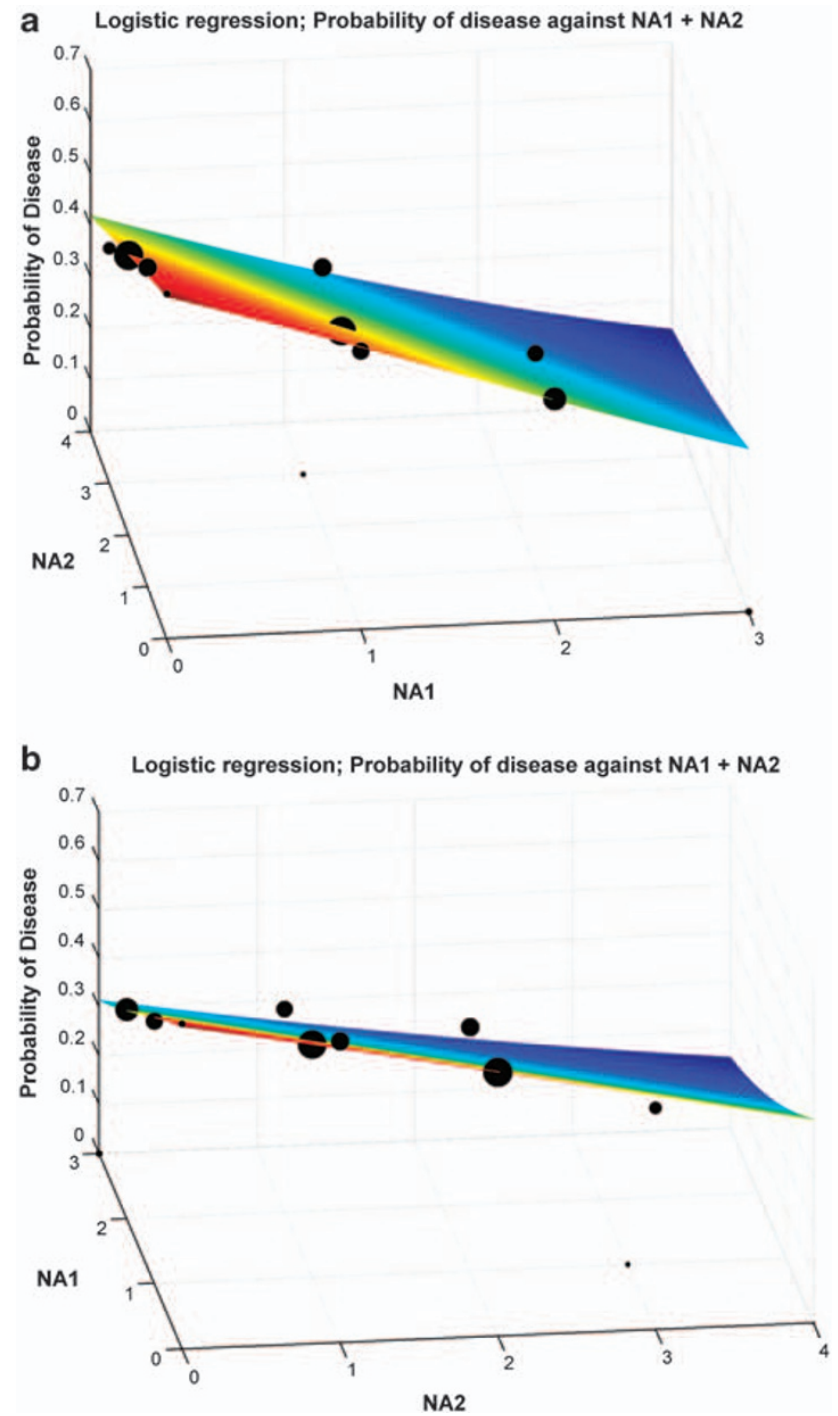

Figure $1 \mathrm{~A}$ graphical representation of a multiple logistic regression fit of disease risk against NA1+NA2. This shows how the probability of disease changes over the different combinations of copies of NA1 and NA2. Black spheres represent data (proportion of individuals with disease for given number of NA1 and NA2), whereas the sizes of the spheres are proportional to the number of samples. The coloured plane represents the model (predicted values). Both plots are of the same data, with (a) being a rotated view from the $x$-axis (NA1) and (b) a reflected view from the NA2 axis.

Table 2 Frequencies of NA1 and total copy number at FCGR3B

\begin{tabular}{lccr}
\hline Variable & Case & Control & P-value \\
\hline NA1 & $0.324(n=723)$ & $0.379(n=749)$ & 0.027 \\
CN & $1.981(n=365)$ & $2.052(n=365)$ & 0.037
\end{tabular}

$P$-values from $t$-test on equal proportions for NA1 and equal means for $\mathrm{CN}$. 
Correlation of FCGR3B CN and NA1/NA2 alleles with SNPs associated with SLE

Missense variants in the genes FCGR2A, FCGR3A and FCGR2B have all been associated with SLE. ${ }^{18,19,26,32}$ Previous estimates of LD between FCGR3B and other FCGR genes would have been inaccurate because of the confounding effect of FCGR3B CNV. Thus, we used our current accurate estimate of FCGR3B CN state and NA1/NA2 allotype to examine LD relations between FCGR3B and other missense polymorphisms that appear to contribute to the risk of SLE potential: FCGR2A-H131R, FCGR3A-F158V and FCGR2B-I232T. Using the data available on the parents from our trio data ${ }^{31}(n=570)$, we calculated (multiple) $R^{2}$ between the alleles at FCGR3B (over all CNs) and the alleles at FCGR2A-H131R, FCGR3A-F158V and FCGR2B-I232T using a regression model, which yielded $0.005(P=0.40), 0.043$ $\left(P=4.35 \times 10^{-8}\right)$ and $0.003 \quad(P=0.37)$, respectively. This agrees very well with the values obtained using a more standard approach $\left(R^{2}\right.$ and $\left.D^{\prime}\right)$ involving all family data with two-copy individuals (156 families), which can be seen in Figure 2. The estimates of $R^{2}$ are small but significant for 3A-F158V, while being extremely small or zero for the others.

The observed correlation between the null haplotype at FCGR3B and these SNPs was even smaller $\left(\max R^{2}=0.01, D^{\prime}=0.56\right.$, not included in figure).

\section{FcyRIIIb expression}

It has been shown previously in one report that FCGR3B CN correlated with cell surface expression. ${ }^{13}$ Given the role of the

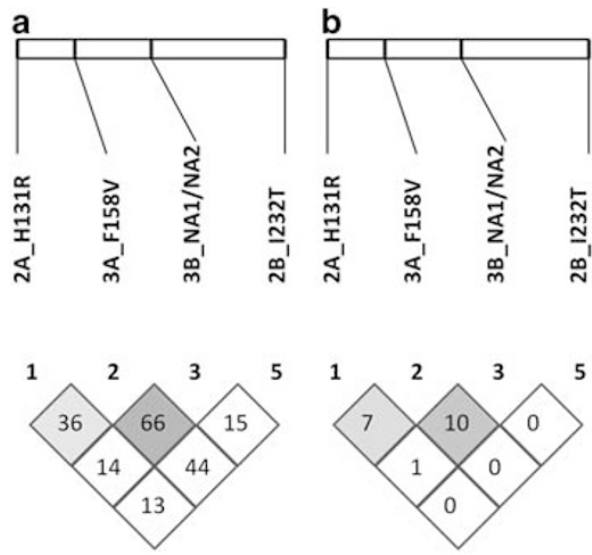

Figure 2 LD plot for surrounding SNPs and two-copy individuals: (a) quantification using $D^{\prime}$, (b) quantification using $R^{2}$.
NA1/NA2 allotype in SLE that we have shown in this paper, we sought to establish whether this allotype affected the expression of the gene product. We observed a positive correlation between neutrophil expression of $\mathrm{F} \gamma \gamma \mathrm{RIIIb}$ (CD16b) as determined by flow cytometry and FCGR3B CN in healthy individuals $\left(n=18 ; R^{2}=0.75 ; P<0.0001\right)$ and individuals with SLE $\left(n=15 ; R^{2}=0.73 ; P<0.0001\right)$. FCGR3B null individuals showed no Fc $\gamma$ RIIIb expression (Figure 3, graphs A and B). Little correlation was observed between neutrophil expression of Fc $\gamma$ RIIIb and NA1/NA2 allotype in two-copy healthy individuals ( $n=26$ ) (Figure 3, graph C). CNV at the FCGR3A locus was controlled and each individual had $\mathrm{CN}=2$.

\section{DISCUSSION}

The results presented here have added to the mounting evidence that a complex and heterogeneous genetic contribution to SLE susceptibility lies within the FCGR region. This work confirms earlier studies that used $\mathrm{qPCR},{ }^{4,13}$ and improves upon them by using the PRT. This is a more reliable and accurate method to determine $\mathrm{CN}$ than $\mathrm{qPCR}$ because of simultaneous amplification of a target and a control. The PRT yields $\mathrm{CN}$ closer to integer values rather than continuous variables as in $\mathrm{QPCR}$, and simultaneously determines relative CNs of the NA1 and NA2 alleles of FCGR3B.

Moreover, our results show that the genetic influence at FCGR3B is complex. The risk of autoimmunity is dependent not only on the number of FCGR3B genes present in the genome but also on the allelic composition of FCGR3B. Specifically, we have shown that the risk of SLE is increased with loss of the higher-affinity ${ }^{33}$ NA1 allele compared with the NA2 allele. Rejection of the $\mathrm{CN}$ model in favour of the biallelic-CN model is interesting, as this suggests that FCGR3B confers risk to SLE as a result of reduced function of Fc $\gamma$ RIIIb on neutrophils, which is secondary to a quantitative effect on gene expression, and a qualitative effect on function, which is secondary to NA1/NA2 allelic composition. We have shown that the SLE genetic association exhibited by FCGR3B cannot be explained by its correlation with other lupus susceptibility alleles at the FCGR locus. A recent study ${ }^{34}$ failed to show an association between NA1/NA2 and SLE; however, in the absence of simultaneous estimation of $\mathrm{CNV}$, it is impossible to establish or refute an effect from NA1/NA2.

It is possible that the large deletion that includes FCGR3B affects the expression of neighbouring genes at the FCGR locus. Given the association of missense alleles at FCGR2A, FCGR3A and FCGR2B, it is possible that altered expression of any of these genes secondary to the FCGR3B deletion event might explain the CN association. However, there are several lines of evidence that indicate that FCGR3B itself underlies the genetic association data. First, it was shown previously
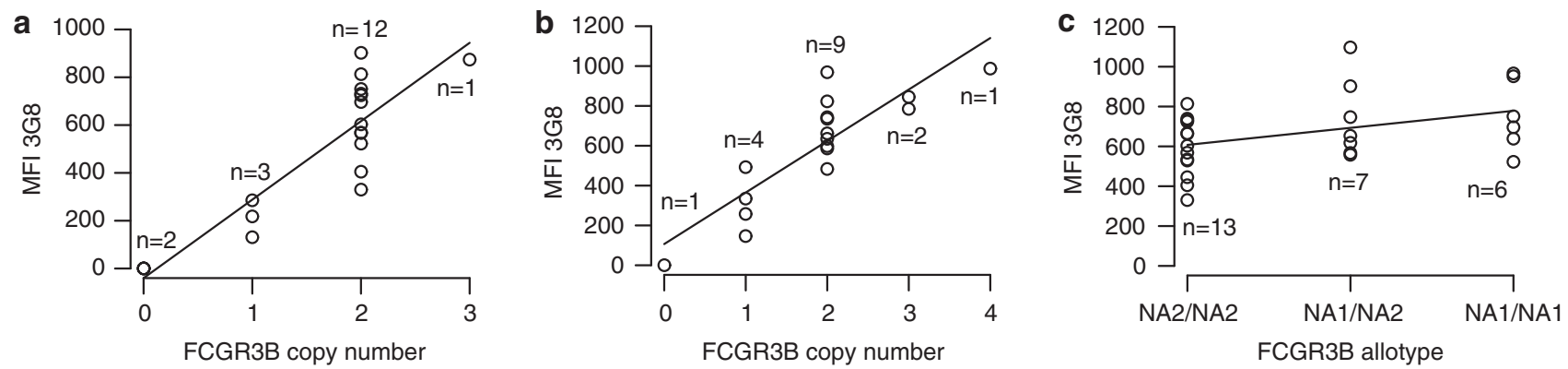

Figure 3 The neutrophil surface expression of Fc $\gamma \mathrm{RIIIb}$ (CD16, clone 3G8) positively correlates with FCGR3B copy number in (a) healthy controls ( $n=18$; $\left.R^{2}=0.75 ; P<0.0001\right)$ and (b) SLE patients $\left(n=15 ; R^{2}=0.73 ; P<0.0001\right)$. (c) Little or no correlation was observed between neutrophil expression of $\mathrm{Fc} \gamma \mathrm{RIIIb}$ and NA1/NA2 allotype in healthy controls with two copies of FCGR3B $\left(n=26 ; R^{2}=0.16 ; P=0.06\right)$. 
that the number of copies of FCGR3B correlated with the magnitude of expression of the gene product on neutrophils. ${ }^{13}$ We replicated these findings, observing a correlation with $\mathrm{CN}$ and neutrophil expression in both healthy controls and patients with SLE $\left(R^{2}=0.75\right)$. Our data showing that NA1 alleles have a greater protective effect in SLE than NA2 alleles further support a role for Fc $\gamma$ RIIIb. As NA1 alleles have a higher affinity for ligand than NA2 alleles, ${ }^{33}$ loss of NA1 should have a greater functional effect than loss of NA2 alleles. We sought to determine whether NA alleles affected Fc $\gamma$ RIIIb expression, and did not observe any marked correlation. This suggests that a functional effect underlies the association found, such as the NA1 allotype harbouring a higher affinity than NA2 for immune complexes containing IgG1 and IgG3. ${ }^{35}$

Our methodology for analysing CNV with family data shows how to make inference on allelic risk when standard methods such as the transmission disequilibrium test cannot be applied. The approach is novel, simple to implement and can be applied using standard statistical software.

The results presented in this paper provide strong evidence that $F C G R 3 B$, a gene solely expressed on neutrophils, is directly involved in the complex genetic process that leads to SLE. This adds weight to a priori beliefs based on the known function of FCGR genes, which identify the region as a disease susceptibility locus. Our approach has unravelled the confounding effect of $\mathrm{CNV}$ on the FCGR3B gene association with SLE and produced evidence of an allelic contribution to disease risk. This raises an important issue of the role of the neutrophil in SLE, a relatively unexplored area of research.

\section{CONFLICT OF INTEREST}

The authors declare no conflict of interest.

\section{ACKNOWLEDGEMENTS}

We thank the study patients and their families for participating in this research, along with clinical colleagues who facilitated data collection. In particular, we thank the Wellcome Trust for funding this work and funding ALR, ASW and $\mathrm{PB}$ and the arc for funding DLM and RT.

1 Rhodes B, Vyse TJ: General aspects of the genetics of SLE. Autoimmunity 2007; 40: 550-559.

2 Vyse TJ: Understanding lupus: fishing genes out of mice and men. Immunity 2008; 28: 8-10.

3 Criswell LA: The genetic contribution to systemic lupus erythematosus. Bull NYU Hosp Jt Dis 2008; 66: 176-183.

4 Fanciulli M, Norsworthy PJ, Petretto E et al: FCGR3B copy number variation is associated with susceptibility to systemic, but not organ-specific, autoimmunity. Nat Genet 2007; 39: 721-723.

5 Shaw-Smith C, Redon R, Rickman L et al: Microarray based comparative genomic hybridisation (array-CGH) detects submicroscopic chromosomal deletions and duplications in patients with learning disability/mental retardation and dysmorphic features. J Med Genet 2004; 41: 241-248.

6 lafrate AJ, Feuk L, Rivera MN et al: Detection of large-scale variation in the human genome. Nat Genet 2004; 36: 949-951.

7 Sebat J, Lakshmi B, Troge J et al: Large-scale copy number polymorphism in the human genome. Science 2004; 305: 525-528.

8 Fanciulli M, Petretto E, Aitman TJ: Gene copy number variation and common human disease. Clin Genet 2009; 77: 201-213.

9 Urban TJ, Weintrob AC, Fellay J et al: CCL3L1 and HIV/AIDS susceptibility. Nat Med 2009; 15: 1110-1112.
10 Field SF, Howson JMM, Maier LM et al: Experimental aspects of copy number variant assays at CCL3L1. Nat Med 2009; 15: 1115-1117.

11 Gonzalez E, Kulkarni $\mathrm{H}$, Bolivar $\mathrm{H}$ et al: The influence of CCL3L1 gene-containing segmental duplications on HIV-1/AIDS susceptibility. Science 2005; 307: 1434-1440.

12 Yang Y, Chung EK, Wu YL et al: Gene copy-number variation and associated polymorphisms of complement component $\mathrm{C} 4$ in human systemic lupus erythematosus (SLE): low copy number is a risk factor for and high copy number is a protective factor against SLE susceptibility in European Americans. Am J Hum Genet 2007; 80: 1037-1054.

13 Willcocks LC, Lyons PA, Clatworthy MR et al: Copy number of FCGR3B, which is associated with systemic lupus erythematosus, correlates with protein expression and immune complex uptake. J Exp Med 2008; 205: 1573-1582.

14 McCarroll SA, Huett A, Kuballa $P$ et al: Deletion polymorphism upstream of IRGM associated with altered IRGM expression and Crohn's disease. Nat Genet 2008; 40: 1107-1112.

15 Breunis WB, van Mirre E, Geissler J et al: Copy number variation at the FCGR locus includes FCGR3A, FCGR2C and FCGR3B but not FCGR2A and FCGR2B. Hum Mutat 2009; 30: 640-650.

16 Karassa FB, Trikalinos TA, Ioannidis JP: Fc gamma RIIIA-SLE meta-analysis investigators. The Fc gamma RIIIA-F158 allele is a risk factor for the development of lupus nephritis: a meta-analysis. Kidney Int 2003; 63: 1475-1482.

17 Kono H, Kyogoku C, Suzuki T et al: FcgammaRIIB Ile232Thr transmembrane polymorphism associated with human systemic lupus erythematosus decreases affinity to lipid rafts and attenuates inhibitory effects on B cell receptor signaling. Hum Mol Genet 2005; 14: 2881-2892.

18 Floto RA, Clatworthy MR, Heilbronn KR et al: Loss of function of a lupus-associated FcgammaRIlb polymorphism through exclusion from lipid rafts. Nat Med 2005; 11: 1056-1058.

19 Brown EE, Edberg JC, Kimberly RP: Fc receptor genes and the systemic lupus erythematosus diathesis. Autoimmunity 2007; 40: 567-581.

20 Yap SN, Phipps ME, Manivasagar M, Bosco JJ: Fc gamma receptor IIIB-NA gene frequencies in patients with systemic lupus erythematosus and healthy individuals of Malay and Chinese ethnicity. Immunol Lett 1999; 68: 295-300.

21 Hatta Y, Tsuchiya N, Ohashi J et al: Association of Fc gamma receptor IIIB, but not of Fc gamma receptor IIA and IIIA polymorphisms with systemic lupus erythematosus in Japanese. Genes Immun 1999; 1: 53-60.

22 González-Escribano MF, Aguilar F, Sánchez-Román J, Núñez-Roldán A: FcgammaRIIA FcgammaRIIIA and FcgammaRIIIB polymorphisms in Spanish patients with systemic lupus erythematosus. Eur J Immunogenet 2002; 29: 301-306.

23 Cukier HN, Pericak-Vance MA, Gilbert JR, Hedges DJ: Sample degradation leads to false-positive copy number variation calls in multiplex real-time polymerase chain reaction assays. Anal Biochem 2009; 386: 288-290.

24 Hollox EJ, Detering J-C, Dehnugara T: An integrated approach for measuring copy number variation at the FCGR3B (CD16) locus. Hum Mut 2009; 30: 477-484.

25 Walker S, Janyakhantikul S, Armour JAL: Multiplex paralogue ratio tests for accurate measurement of multiallelic CNVs. Genomics 2009; 93: 98-103.

26 Karassa FB, Trikalinos TA, Ioannidis JP: Fc gamma RIIIA-SLE meta-analysis investigators. The Fc gamma RIIIA-F158 allele is a risk factor for the development of lupus nephritis: a meta-analysis. Kidney Int 2003; 63: 1475-1482.

27 Tan EM, Cohen AS, Fries JF et al: The 1982 revised criteria for the classification of systemic lupus erythematosus. Arthritis Rheum 1982; 25: 1271-1277.

28 Zhou P, Comenzo RL, Olshen AB et al: CD32B is highly expressed on clonal plasma cells from patients with systemic light-chain amyloidosis and provides a target for monoclonal antibody-based therapy. Blood 2008; 111: 3403-3406.

29 Spielman RS, McGinnis RE, Ewens WJ: Transmission test for linkage disequilibrium: the insulin gene region and insulin-dependent diabetes mellitus (IDDM). Am J Hum Genet 1993; 52: 506-516.

30 Lehmann EL: Testing Statistical Hypotheses, 2nd edn. New York: John Wiley and Sons, 1986.

31 Roberton CA, Morley BJ, Vyse TJ: Fine mapping genes for Fc gamma receptor II and III in systemic lupus erythematosus. Am J Hum Genet 2001; 69: 568

32 Kono H, Kyogoku C, Suzuki T et al: FcgammaRIIB Ile232Thr transmembrane polymorphism associated with human systemic lupus erythematosus decreases affinity to lipid rafts and attenuates inhibitory effects on B cell receptor signaling. Hum Mol Genet 2005: 14: 2881-2892.

33 Salmon JE, Edberg JC, Kimberly RP: Fc gamma receptor III on human neutrophils. Allelic variants have functionally distinct capacities. J Clin Invest 1990; 85: 1287-1295.

34 Lee YH, Ji JD, Song GG: Fc $\gamma$ receptor IIB and IIIB polymorphisms and susceptibility to systemic lupus erythematosus and lupus nephritis: a meta-analysis. Lupus 2009; 18 : 727-734.

35 Bredius RG, Fijen CA, De Haas M: Role of neutrophil Fc gamma Rlla (CD32) and Fc gamma RIIIb (CD16) polymorphic forms in phagocytosis of human IgG1 and IgG3opsonized bacteria erythrocytes. Immunology 1994; 83: 624-630.

Supplementary Information accompanies the paper on European Journal of Human Genetics website (http://www.nature.com/ejhg) 